Article

\title{
Thymoquinone-Loaded Chitosan Nanoparticles as Natural Preservative Agent in Cosmetic Products
}

\author{
María Mondéjar-López ${ }^{1}$, Alberto José López-Jiménez ${ }^{1}$, Joaquín C. García Martínez ${ }^{2,3}$ 이 , Oussama Ahrazem ${ }^{1}($, \\ Lourdes Gómez-Gómez ${ }^{1}$ (D) and Enrique Niza ${ }^{1, *}$ (D) \\ 1 Instituto Botánico, Departamento de Ciencia y Tecnología Agroforestal y Genética, Universidad de Castilla-La \\ Mancha, Campus Universitario s/n, 02071 Albacete, Spain; maria.mondejar3@alu.uclm.es (M.M.-L.); \\ albertojose.lopez@uclm.es (A.J.L.-J.); Oussama.ahrazem@uclm.es (O.A.); \\ MariaLourdes.gomez@uclm.es (L.G.-G.) \\ 2 Departamento de Química Inorgánica, Orgánica y Bioquímica, Facultad de Farmacia, Universidad de \\ Castilla-La Mancha, C/José María Sánchez Ibáñez s/n, 02008 Albacete, Spain; Joaquinc.garcia@uclm.es \\ 3 Regional Center for Biomedical Research (CRIB), Universidad de Castilla-La Mancha, C/Almansa 13, \\ 02008 Albacete, Spain \\ * Correspondence: enrique.niza@uclm.es
}

Citation: Mondéjar-López, M.; López-Jiménez, A.J.; Martínez, J.C.G.; Ahrazem, O.; Gómez-Gómez, L.; Niza, E. Thymoquinone-Loaded Chitosan Nanoparticles as Natural Preservative Agent in Cosmetic Products. Int. J. Mol. Sci. 2022, 23, 898. https://doi.org/10.3390/ ijms23020898

Academic Editors: Monica Terracciano, Ilaria Rea, Nicola Borbone and Chiara Tramontano

Received: 23 December 2021

Accepted: 13 January 2022

Published: 14 January 2022

Publisher's Note: MDPI stays neutral with regard to jurisdictional claims in published maps and institutional affiliations.

Copyright: (C) 2022 by the authors. Licensee MDPI, Basel, Switzerland. This article is an open access article distributed under the terms and conditions of the Creative Commons Attribution (CC BY) license (https:// creativecommons.org/licenses/by/ $4.0 /)$.

\begin{abstract}
The current status of controversy regarding the use of certain preservatives in cosmetic products makes it necessary to seek new ecological alternatives that are free of adverse effects on users. In our study, the natural terpene thymoquinone was encapsulated in chitosan nanoparticles. The nanoparticles were characterized by DLS and TEM, showing a particle size of $20 \mathrm{~nm}$. The chemical structure, thermal properties, and release profile of thymoquinone were evaluated and showed a successful stabilization and sustained release of terpenes. The antimicrobial properties of the nanoparticles were evaluated against typical microbial contaminants found in cosmetic products, showing high antimicrobial properties. Furthermore, natural moisturizing cream inoculated with the aforementioned microorganisms was formulated with thymoquinone-chitosan nanoparticles to evaluate the preservative efficiency, indicating its promising use as a preservative in cosmetics.
\end{abstract}

Keywords: nanotechnology; cosmetic; chitosan; essential oils; antimicrobial; preservative agents; nanoparticles

\section{Introduction}

Microorganism contaminants are one of the most usual causes of alteration in cosmetic products (CPs) due to over-exposition to atmospheric oxygen [1]. When the CPs are opened for daily use, the microorganisms present in the atmosphere make contact with the cosmetic formulation, producing problems such as the appearance of mold, separation of phases of the emulsions, loss of viscosity, change in aroma, or rancidity of fats [2]. Moreover, the presence of pathogenic microorganisms in CPs has been associated with the development of some adverse effects, such as dermatitis, irritation, or peeling as well as conjunctivitis, asthma, urticaria, angioedema, or pneumonia [3].

The rising CP market, which is projected to reach $\$ 429.8$ billion by 2022 , is linked to an increased consumption of long shelf-life products. The cosmetic industry uses several compounds to prolong the period of use in cosmetics. One of the most common approaches is the incorporation of preservative agents, i.e., substances that inhibit the growth of microorganisms and counteract the generation of reactive oxygen species and oxidation processes [4]. Some cosmetic preservatives such as parabens, triclosan, benzalkonium chloride, formaldehyde, phenoxyethanol, and chlorphenesin produce adverse effects in humans such as DNA damage, antiandrogenic activity, cytotoxicity and genotoxic effects on human lymphocytes, cytotoxicity in meibomian glands, risk of cancer, and allergic reactions and also produce environmental and animal toxicity [5,6]. Due to these results, 
the traditional use of preservatives in cosmetics is leading us to search for new "eco-friendly" and efficient alternatives.

Essential oils (EO) are volatile compounds present in some aromatic plants and spices. Terpenes and isoprenoids are compounds derived from isoprenes and are the main component of several EOs that are widely used in medicine due to their varied biological and pharmacological activity, including high antimicrobial and antioxidant properties [7]. Thymoquinone (TQ) is the most abundant constituent of the volatile oil of Nigella sativa seeds, contributing to most of the properties of $N$. sativa. Different pharmacological properties of TQ have been previously described, e.g., immunomodulatory [8], anti-histaminic [9], anti-tumor [10], hepatoprotective [11], gastroprotective [12], nephroprotective [13], neuroprotective [14], antioxidant [15], and antimicrobial activity [16]. However, its high volatility and easy degradation can make its applications difficult.

The encapsulation of terpenes in different raw materials can improve the stability of compounds and increase their therapeutic efficacy. One of the most promising approaches is through the use of nanotechnology, with the encapsulation of terpenes in different formulations with a range of sizes between 1-1000 nm, thus improving their effect and stability [17].

Chitosan $(\mathrm{CH})$, a linear polysaccharide consisting of 1, 4-linked glucosamine and $\mathrm{N}$-acetylglucosamine, is a natural polymer derived through deacetylation of chitin in an alkaline media, with chitosan being the major component in shrimp and other crustaceans ${ }^{\prime}$ cuticular exoskeletons [18]. CH is one of the most popular polysaccharides used as a raw material to encapsulate active compounds such as essential oils, terpenes, and several drugs due to its structure-forming, biocompatibility, and high stability $[19,20]$. Due to its versatile and easy manipulation, several formulations of chitosan have been formulated such as gel beads [21], polyelectrolyte complex-based hydrogels [22], microparticles [23], and nanoparticles, among others. The encapsulation of TQ into chitosan nanoparticles has been tested in different medical areas [14], where the TQ encapsulation is evaluated in radioiodinated folic acid-chitosan nanoparticles in order to target ovarian cancer, showing lower cytotoxicity in healthy cells [24]. Encapsulation of TQ in chitosan nanoparticles coated with polysorbate 80 showed an improvement in the antidepressant effect of terpene in Wistar rodents [25]. Moreover, in the cosmetic industry, $\mathrm{CH}$ is widely applied in CPs as an antioxidant, emulsifying agent, and skin protective agent in chitosan form or in different chitosan forms such as carboxymethyl chitosan [26].

Thus, the present study shows, for the first time, the assessment of TQ encapsulated in chitosan nanoparticles (NPCH-TQ) as a cosmetic preservative agent through the evaluation of antimicrobial activity in natural formulated moisturizing cream.

\section{Results}

\subsection{Particle Size and Z Potential of NPCH and NPCH-TQ}

Photon correlation spectroscopy or dynamic light scattering (DLS) is a technique based on the scattering of a laser beam of a given wavelength by particles or macromolecules in a liquid medium due to their Brownian motion, which is widely used to determine particle size and surface charge. The results presented in Table 1 show an average range of nanoparticle size from 48.6 to $65.0 \mathrm{~nm}$, showing an increase in size from non-encapsulated nanoparticles due to the incorporation of terpenes into raw material. The results of the polydispersity index (PDI) show values below 0.5, achieving PDI values of 0.2 in the formulations of ratios 1:0.75 and 1:1. All nano-formulations present positive $Z$ values from +23.9 up to $+49.8 \mathrm{mV}$ due to the use of chitosan as a raw material. The results presented in Table 1 show a decreasing trend in $\mathrm{Z}$ values related to the amount of TQ encapsulated in nanoparticles. 
Table 1. DLS measures, encapsulation efficiency (EE\%), and efficient loading (EL\%) of nano-formulations.

\begin{tabular}{cccccc}
\hline Formulation & Average Size (nm) & PDI & Z-Value (mV) & EE\% & EL\% \\
\hline NPCH & $48.6 \pm 3.40$ & $0.4 \pm 0.02$ & $+49.8 \pm 0.75$ & - & - \\
NPCH-TQ 1:0.25 & $65.0 \pm 1.40$ & $0.4 \pm 0.01$ & $+35.8 \pm 3.23$ & $88.2 \pm 6.39$ & $44.8 \pm 0.70$ \\
NPCH-TQ 1:0.5 & $57.5 \pm 0.36$ & $0.3 \pm 0.01$ & $+27.8 \pm 1.13$ & $93.2 \pm 2.05$ & $48.4 \pm 1.14$ \\
NPCH-TQ 1:0.75 & $57.5 \pm 0.33$ & $0.2 \pm 0.01$ & $+25.3 \pm 0.76$ & $94.5 \pm 1.94$ & $48.8 \pm 1.24$ \\
NPCH-TQ 1:1 & $63.4 \pm 0.65$ & $0.2 \pm 0.01$ & $+23.9 \pm 0.58$ & $90.6 \pm 10.4$ & $50.7 \pm 8.70$ \\
\hline
\end{tabular}

\subsection{Successful Encapsulation of Thymoquinone in Chitosan Nanoparticles}

Encapsulation efficiency and efficient loading are parameters related to the capacity of encapsulation of drugs by different raw materials and the quality of formulation. These parameters mainly depend on the type of raw material, drug polarity, and approach used in nanoparticle preparation. The results presented in Table 1 show different EE\% and EL\% obtained by the different ratios used to obtain NPCH-TQ. The EE\% values are between $88.2 \%$ and $94.5 \%$, showing the maximum value in the 1:0.75 ratio. The maximum EL\% value reached $50.7 \%$ in the $1: 1$ ratio corresponding to the formulation with the highest amount of initial thymoquinone used.

\subsection{Morphology Characterization of Nanoparticles}

Transmission Electron Microscope (TEM) is a common microscope technique to determine the surface properties and morphology characteristics of nanoparticles and nanoformulations [27]. Figure 1 shows two different micrographs which correspond to (A) $\mathrm{NPCH}$ and (B) NPCH-TQ 1:1. Both images show nanoparticles with non-defined surfaces, clearly trending toward forming aggregates or gel-like structures. All nanoparticles have a size smaller than $50 \mathrm{~nm}$ with approximately $20 \mathrm{~nm}$ with narrow size distribution.
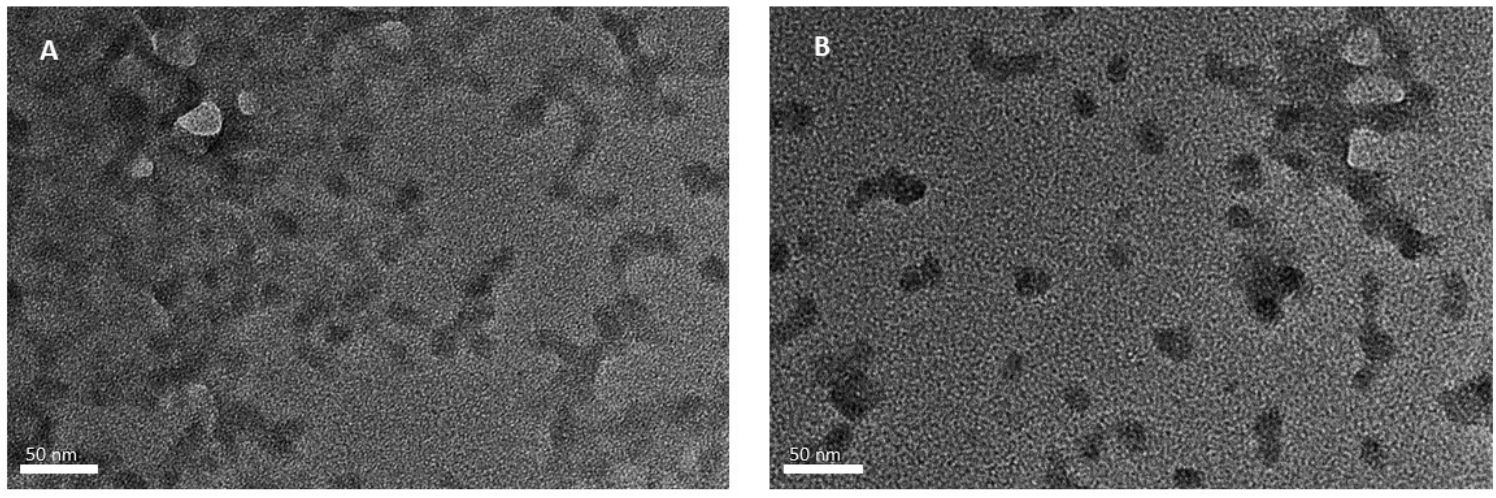

Figure 1. TEM micrograph of (A) NPCH and (B) NPCH-TQ 1:1.

\subsection{Chemical Structure of NPCH-TQ}

The chemical composition of NPCH-TQ was determined by identification of the principal functional groups through the FTIR technique. The attenuated total reflectance (ATR) accessory allowed us to identify the main functional groups present directly from the nanoparticles or essential oils without the need to use matrices. FTIR is typically employed to investigate the interaction between functional groups. However, this technique allows us to verify the existence of functional groups whose vibrational modes are characteristic. FTIR spectra of TQ, NPCH, and NPCH-TQ are shown in Figure S1. In the TQ spectrum, the signals corresponding to the stretching vibrations of the most important functional groups are observed. The most intense signal in the spectrum is broadband at $1636 \mathrm{~cm}^{-1}$, which corresponds to the overlap of two vibrations, the $\mathrm{C}=\mathrm{O}$ double bond strain and the $\mathrm{C}=\mathrm{C}$ double bond strain. The conjugate system between the $C=O$ and $C=C$ of the TQ makes these vibrations overlap in the spectrum. Moreover, intense bands corresponding to the $\mathrm{sp}^{3}$ car- 
bon C-H bonds $\left(2969 \mathrm{~cm}^{-1}\right)$ and the C-O bond strain $\left(1249 \mathrm{~cm}^{-1}\right)$ are observed. The NPCH and NPCH-TQ spectra are very similar since their content is mainly chitosan. A broadband between $3750 \mathrm{~cm}^{-1}$ and $2500 \mathrm{~cm}^{-1}$, associated with the stretch of the $\mathrm{O}-\mathrm{H}$ and N-H bonds, was clearly observed. As shown in the gravimetric analysis, chitosan nanoparticles were hygroscopic and had a great tendency to absorb water. This signal includes both chitosan $\mathrm{O}-\mathrm{H}$ structure and water in its different strengths due to the hydrogen bonds formed. From the spectra, additional characteristic peaks of chitosan were observed at $2916 \mathrm{~cm}^{-1}$ and $2864 \mathrm{~cm}^{-1}$ due to $\mathrm{C}-\mathrm{H}$ bond stretching of sp $\mathrm{sp}^{3}$ carbon, $1634 \mathrm{~cm}^{-1}$ due to carbonyl stretching $\mathrm{C}=\mathrm{O}$ of the amide, $1533 \mathrm{~cm}^{-1}$ due to $\mathrm{N}-\mathrm{H}$ bending of the chitosan, and $1066 \mathrm{~cm}^{-1}$ due to the tension of the different $\mathrm{C}-\mathrm{O}$ bonds. Because the most important $\mathrm{TQ}$ signals overlap with important bands in the $\mathrm{NPCH}$, it is difficult to appreciate significant changes, although variations in signal intensities indicate the presence of TQ in the nanoparticles [28].

\subsection{Thermal Properties of NPCH-TQ}

Thermo Gravimetric Analysis/Differential Scanning Calorimetry (TGA/DSC) was used to evaluate the absorbed material, thermal stability, and decomposition temperatures of the TQ, NPCH, and NPCH-TQ. TQ is a volatile yellowish solid. Figure S1a shows the combination of TGA and DSC for TQ. In DSC, an endothermic peak is observed at $45{ }^{\circ} \mathrm{C}$ $(119 \mathrm{~J} / \mathrm{g})$ which correlates with the loss of virtually all mass in TGA. This indicates that the compound is volatile and sublimes at this temperature. Previously, we showed that chitosan nanoparticles have two degradation steps, the initial step starting from the very beginning of the experiment and ending at $100{ }^{\circ} \mathrm{C}$, and the second one starting at $170{ }^{\circ} \mathrm{C}$ up to $333^{\circ} \mathrm{C}$ (Figure S2). The first transition corresponds to a 3\% mass drop and is attributed to the loss of adsorbed/bound water/moisture vaporization [28]. The degradation of the pure chitosan biopolymer resulted in a $60 \%$ weight loss in the second stage. This has been found previously for various chitosan polymers, where the amount of moisture and the range of breakdown temperatures are dependent on the chitosan polymer's molecular weight. A similar trend is observed in the NPCH-TQ thermogram (Figure S2b), coinciding in the temperature ranges. In the second transition, the mass loss was 58\%, matching with the degree of NPCH decomposition [28]. In first transition, the mass loss is considerably higher, reaching $9.4 \%$ and, even though the water absorbed by both particles may differ, this may indicate that this $9.4 \%$ loss could correspond not only to water but also to TQ loss. These data were confirmed by comparing the endothermic peaks between $25^{\circ} \mathrm{C}$ and $100{ }^{\circ} \mathrm{C}$. There is a considerable difference between the NPCH and NPCH-TQ evaporation enthalpies. For NPCH, an enthalpy of $145 \mathrm{~J} / \mathrm{g}$ has been determined, while NPCH-TQ shows a higher value, reaching $274 \mathrm{~J} / \mathrm{g}$, which indicates that TQ-loaded nanoparticles need more energy to evaporate both water and TQ.

\subsection{In Vitro Drug Release of NPCH-TQ}

Drug release studies can offer information concerning nanoparticle behavior to release its encapsulated drugs. In this study, the $\mathrm{pH}$ of the buffer release medium was chosen according to the nanoparticle application. In this case, the $\mathrm{pH}$ of the CPs was close to 6 . The results presented in Figure 2 show a triphasic release profile of $T Q$, achieving a maximum of $62 \%$ of TQ release at 28 days. Nanoparticles showed a slow initial release reaching $11 \%$ TQ in the first $24 \mathrm{~h}$. Then, the release of TQ was governed by diffusion and degradation of the polymer matrix showing a slow-release profile due to the high hydrophobicity of $T Q$, resulting in the release mechanism being dependent on the nature of the raw material. 


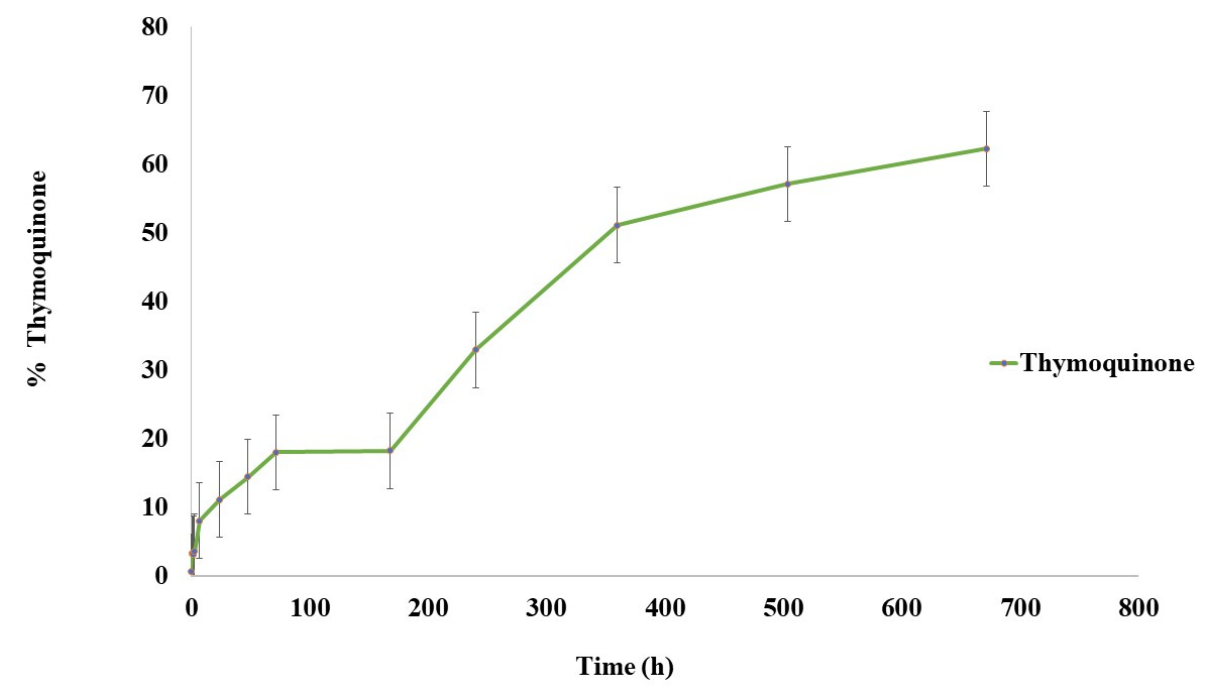

Figure 2. In vitro drug release of NPCH-TQ in PBS at $\mathrm{pH} 6$.

\subsection{DPPH Scavenging Activity of Free TQ and Nano-Formulations}

To determine the antioxidant properties of nano-formulations, we used the DPPH scavenging method. DPPH was chosen for its simplicity since it is one of the few organic radicals with nitrogen atoms in its structure. This structure confers stability because of the delocalization of an unpaired electron on the molecule. This delocalization also causes an intensification in the purplish color characteristic of the radical, which in an ethanolic medium absorbs at $515 \mathrm{~nm}$. This purplish coloration of the solution is attenuated in the presence of an antioxidant that can donate or transfer a hydrogen atom, giving a yellowish color due to the reduced form of DPHP-H [5]. Figure 3 presents the \% of DPPH scavenging of free TQ, NPCH and NPCH-TQ on the range of concentrations between 0.125 and 1 of tested material. NPCH did not show antioxidant properties against DPPH in the concentration range tested. Free TQ shows a maximum of $83.6 \%$ of inhibition of DPPH at $1 \mathrm{mg} / \mathrm{mL}$ of TQ, higher than NPCH-TQ, which showed a maximum inhibition of $29.3 \%$ at $1 \mathrm{mg} / \mathrm{mL}$ of total material corresponding to $0.48 \mathrm{mg} / \mathrm{mL}$ of pure TQ.

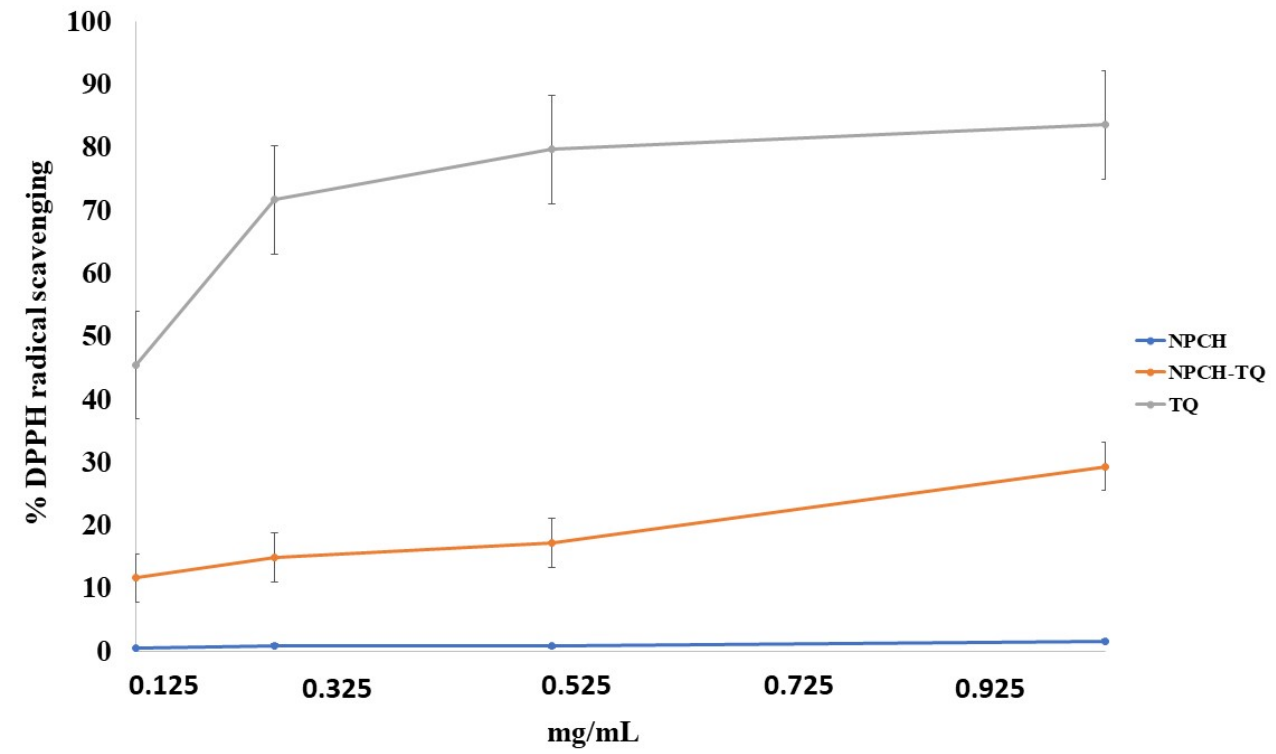

Figure 3. DPPH scavenging of free TQ, NPCH, and NPCH-TQ. 


\subsection{Antimicrobial Evaluation of Free Terpene and Its Nano-Formulation}

The microorganisms used to check the activity in preservative agents in CPs are $P$. aeruginosa, E. coli, S. aureus, $A$. brasiliensis, and $C$. albicans. The broth microdilution method is a widely used technique to evaluate the antimicrobial properties of volatile compounds such as essential oil and terpenes [17,29]. The Minimum Inhibitory Concentrations (MICs) after $24 \mathrm{~h}$ of treatment for bacteria and $48 \mathrm{~h}$ for fungi are presented in Table 2. The minimum inhibitory concentrations $(\mu \mathrm{g} / \mathrm{mL})$ of different treatments are presented in Table 2. NPCH did not display an increase in the antimicrobial effect with respect to the control treatments against tested microorganisms, showing the same MICs in E. coli and P. aeruginosa $(1000 \mu \mathrm{g} / \mathrm{mL})$ and lower MICS against S. aureus, C. albicans, and A. brasiliensis $(>1000 \mu \mathrm{g} / \mathrm{mL})$. The encapsulation of TQ in chitosan nanoparticles resulted in improved antimicrobial activity reaching $292 \mu \mathrm{g} / \mathrm{mL}, 417 \mu \mathrm{g} / \mathrm{mL}$, and $333 \mu \mathrm{g} / \mathrm{mL}$ in E. coli, P. aeruginosa, and S. aureus, respectively. However, the NPCH-TQ showed lower MICs than free TQ against $C$. albicans and $A$. brasiliensis, obtaining $250 \mu \mathrm{g} / \mathrm{mL}$ and $500 \mu \mathrm{g} / \mathrm{mL}$, respectively.

Table 2. Minimum inhibitory concentrations $(\mu \mathrm{g} / \mathrm{mL})$ of control drugs (Gentamicin $\left(^{*}\right)$ and Tebuconazole $\left.{ }^{* *}\right)$ ), TQ, NPCH, and NPCH-TQ.

\begin{tabular}{ccccc}
\hline Microorganism & $\begin{array}{c}\text { Control MIC } \\
(\mu \mathrm{g} / \mathrm{mL})\end{array}$ & TQ $(\mu \mathrm{g} / \mathrm{mL})$ & NPCH $(\mu \mathrm{g} / \mathrm{mL})$ & $\begin{array}{c}\text { NPCH-TQ } \\
(\mu \mathrm{g} / \mathrm{mL})\end{array}$ \\
\hline E. coli & $* 1000$ & 1000 & 1000 & 292 \\
P. aeruginosa & $* 1000$ & $>1000$ & 1000 & 417 \\
S. aureus & $* 1000$ & $>1000$ & $>1000$ & 333 \\
C. albicans & $* * 250$ & 333 & $>1000$ & 250 \\
A. brasiliensis & $* * 250$ & 250 & $>1000$ & 500 \\
\hline
\end{tabular}

\subsection{Evaluation of NPCH-TQ as a New Preservative Agent in Cosmetic Products}

In order to evaluate the effect of NPCH-TQ as new a preservative agent in cosmetic products, we formulated a natural moisturizing cream with the ingredients shown in Table 3. After formulation, control cream and NPCH-TQ cream were both inoculated with common contaminant microorganisms in CPs as recommended by UNI EN ISO 11930:2012, detailed in Section 4.8. At different intervals of days, $1 \mathrm{~g}$ of the creams was inoculated in different culture media, and the UFC/g was counted for each microorganism. The results of the antimicrobial activity of NPCH-TQ in the formulated cream are presented in Figure 4. The control cream did not display any preservative activity against all tested microorganisms, showing an increasing trend over the days in all bacteria and fungi reaching $10^{8} \mathrm{UFC} / \mathrm{gr}$ for $P$. aeruginosa and E. coli and $10^{7}$ for S. aureus. Preserved cream with NPCH-TQ displayed high antibacterial activity against E. coli and $S$. aureus, reducing the UFC/g to zero. Meanwhile, in P. aeruginosa, NPCH-TQ showed bacteriostatic properties during the time of the experiment, maintaining stable CFU/g. The antifungal effects of NPCH-TQ in formulated cream against $A$. brasiliensis and C. albicans are shown in Figure 4 revealed a total reduction in the number of $\mathrm{UFC} / \mathrm{g}$ to zero. 
Table 3. Cream composition. A (Aqueous phase), B (Oil phase), and C (thermolabile compounds).

\begin{tabular}{ccc}
\hline Ingredient & Control Cream (\%) & NPCH-TQ Cream (\%) \\
\hline Water (A) & 47 & 46.5 \\
Vegetable glycerin (A) & 10 & 10 \\
Urea (A) & 3 & 3 \\
\hline Glyceryl monostearate (B) & 8 & 8 \\
Argania spinosa kernel oil (B) & 28 & 28 \\
\hline Allantoin (C) & 0.4 & 0.4 \\
Avena sativa extract (C) & 3 & 3 \\
Vitamin E (C) & 0.5 & 0.5 \\
Parfum (C) & 0.1 & 0.1 \\
NPCH-TQ (C) & - & 0.5 \\
\hline
\end{tabular}

P. aeruginosa

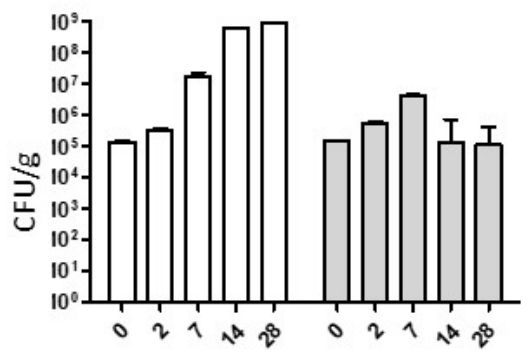

A. brasilensis

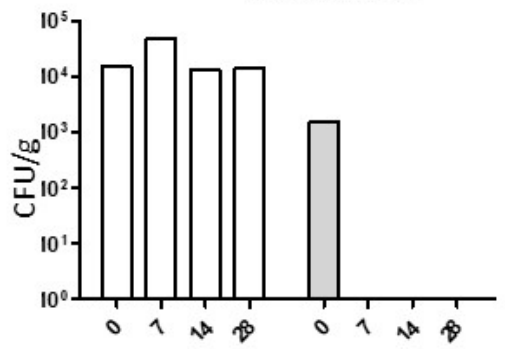

E. coli

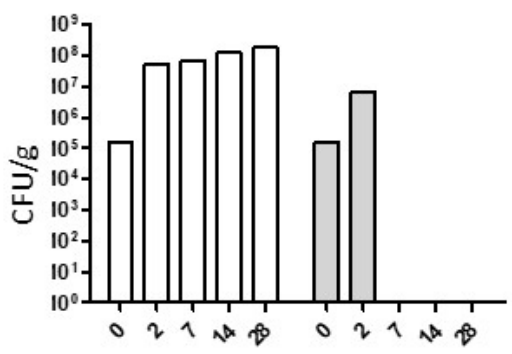

C. albicans

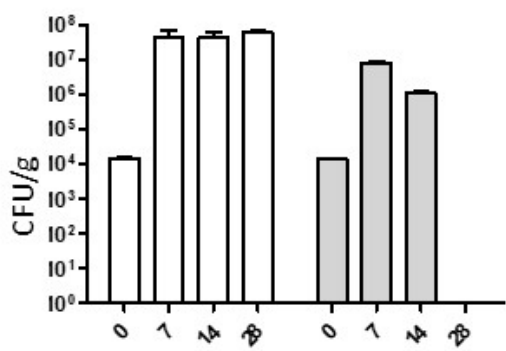

S. aureus

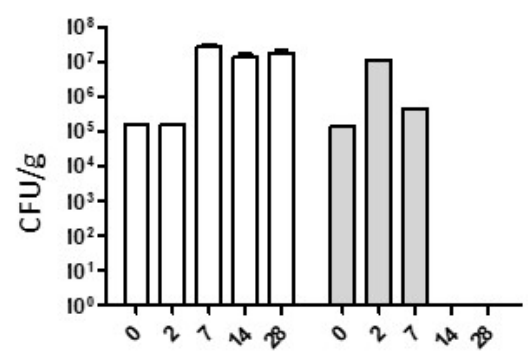

$\square$ Control-Cream

$\square \mathrm{NPCH}-\mathrm{TQ}-\mathrm{Cream}$

Figure 4. Preservative activity of control cream vs. NPCH-TQ.

\section{Discussion}

The main objective of this work is to develop a new alternative to conventional preservative agents based on green nanotechnology through the encapsulation of the natural terpene thymoquinone in chitosan nanoparticles. There is a lack of studies on TQ encapsulation in chitosan formulations; studies have focused mainly on the treatment of breast adenocarcinoma [30], ovarian cancer [24], nose-to-brain targeting [31], and depression treatments [25], among others.

In this study, we obtained four types of nano-formulations of NPCH-TQ through the utilization of different ratios of NPCH:TQ, where the particle size measured by the DLS technique did not show too many differences among obtained nano-formulations. The particle size ranged from 48.6 to $65.0 \mathrm{~nm}$. However, the TEM micrograph presented in Figure 1 shows nanoparticles with a size close to $20 \mathrm{~nm}$. Other works obtained the same trend with terpenes encapsulated in chitosan nanoparticles, obtaining different data regarding particle sizes in DLS and TEM. This was the case in the study performed by Woranuch et al., where chitosan-eugenol nanoparticles showed an average particle size of $683 \mathrm{~nm}$ by DLS and close to $100 \mathrm{~nm}$ by TEM due to the swelling and aggregation effect of chitosan in water solutions [32]. All NPCH-TQ revealed a positive surface charge with a decreasing trend related to the incorporation of TQ into the polymer matrix. The same 
pattern is shown in the encapsulation of other volatile compounds such as garlic essential oil in chitosan nanoparticles with a $\mathrm{Z}$ potential range from +45.8 to $+19.8 \mathrm{mV}$ [28]. The positive surface charge of nanoparticles is associated with increased antimicrobial activity due to their ease of interaction with the wall of microorganisms [17]. Our nano-formulations showed a successful encapsulation of TQ in all tested ratios, with a maximum of $94.5 \%$ of $\mathrm{EE} \%$ and $50.7 \%$ of EL for the ratio 1:1. Other works with chitosan-TQ nanoparticles coated with polysorbate 80 reported an EE\% of $85.6 \%$ and $16.26 \%$ of EL\% [25]. The encapsulation of TQ has also been explored in other polymeric raw materials such as PLGA, showing $\mathrm{EE} \%$ values of $79.9 \%$ [33].

The release profile in long-term experiments gives us an idea of how the nanoparticles release the encapsulated compound with a suitable $\mathrm{pH}$ for $\mathrm{CPs}$ as well as the release profile during 28 days of storage. NPCH-TQ showed a triphasic release profile with a maximum release of $62 \%$ at 28 days. The insolubility of TQ in water results in a slow burst release and diffusion phase showing a slower release profile than other water-soluble drugs such as doxorubicin encapsulated in chitosan nanoparticles, which reached $100 \%$ of release at $170 \mathrm{~h} \mathrm{[34]}$. Another work in which TQ was encapsulated in pegylated polylactide co-glycolide nanoparticles (TF-PEG-PLGA-TQ NPs) for the treatment of non-small cell lung carcinoma achieved a maximum of $50 \%$ cumulative release of TQ in $24 \mathrm{~h}$ at $\mathrm{pH}$ 7.4, showing a faster release than NPCH-TQ due to the more hydrophilic nature of PEG-PLGA, facilitating the release of the hydrophobic compound from raw materials [35]. More hydrophilic terpenes, such as eugenol, showed a faster release than the TQ profile encapsulated in chitosan nanoparticles, with a maximum release of $73 \%$ at $36 \mathrm{~h} \mathrm{[36].}$

The study of antioxidant properties of NPCH-TQ is suitable to increase the usefulness of this nano-formulation in CPs. NPCH-TQ showed a maximum DPPH scavenging of $29.3 \%$ at $1 \mathrm{mg} / \mathrm{mL}$ of total material, lower than other essential oil encapsulated in chitosan nanoparticles such as clove essential oil, which showed a maximum value of $71 \%$ at the same concentration. However, this low value might be due to the fact that at DPPH scavenging measure time ( $30 \mathrm{~min}$ ) and based on the release profile shown in Figure 2, the $\mathrm{TQ}$ released to the medium only reached $3.3 \%$ of total encapsulated TQ, and the antioxidant properties were due mainly to the presence of TQ and not to the chitosan as shown in Figure 3. Another nano-formulation with eugenol encapsulated in chitosan nanoparticles showed a maximum of $70 \%$ inhibition of DPPH at $4.0 \mathrm{mg} / \mathrm{mL}$. Meanwhile, carvacrol encapsulated in the same nano-formulation achieved close to $40 \%$ of DPPH inhibition [37].

The encapsulation of TQ in chitosan nanoparticles resulted in an improvement of antibacterial activity in the bacteria tested. The antibacterial activity of NPCH-TQ was higher against E. coli than S. aureus and P. aeruginosa with $292 \mu \mathrm{g} / \mathrm{mL}, 333 \mu \mathrm{g} / \mathrm{mL}$, and $417 \mu \mathrm{g} / \mathrm{mL}$, respectively. NPCH-TQ displayed a higher antibacterial activity against E. coli and S. aureus than other nanoparticles made of carvacrol and eugenol-chitosan nanoparticles, which showed MICs values of $0.5-1 \mu \mathrm{g} / \mathrm{mL}$ for the same bacteria [37]. However, the encapsulation of TQ in chitosan nanoparticles was not associated with an increase in the antifungal activity of TQ. TQ nanoparticles obtained through the ball milling method obtained lower MICs with values of $160 \mu \mathrm{g} / \mathrm{mL}$ against $C$. albicans [38]. The same trend was observed in A. brasiliensis after NPCH-TQ with an MIC value of $500 \mu \mathrm{g} / \mathrm{mL}$. Garlic essential oil encapsulated in chitosan-TPP nanoparticles via crosslinking showed different MIC values against two different Aspergillus species, A. niger and A. versicolor, with $0.37 \mu \mathrm{g} / \mathrm{mL}$ and $3.33 \mu \mathrm{g} / \mathrm{mL}$, respectively [28].

To confirm the preservative effect of NPCH-TQ in naturally formulated cream, we evaluate the antimicrobial activity in contaminated cream with the microorganisms described above. After 28 days of storage, non-preserved cream (control cream) shows an increase in total UFC/gr in all tested microorganisms, reaching an increase of 3 logs in E. coli, P. aeruginosa, and A. brasiliensis. However, the synthesized NPCH-TQ displayed a total reduction in UFC/gr in E. coli, S. aureus, C. albicans, and A. brasiliensis and a maintenance of the total UFC/gr of P. aeruginosa related to the lower activity of NPCH-TQ against this microorganism. These results confirm the preservative activity of NPCH-TQ in formulated 
creams. Other works with biogenic silver nanoparticles from Iris tuberosa aqueous extract confirm the role of nanotechnology to be used as a platform to obtain new preservative agents [5].

In conclusion, the results shown in this work present a new and effective preservative agent based on the encapsulation of a terpene in chitosan nanoparticles to satisfy the regulations and the growing demands of the consumers of natural, green, and sustainable products. This approach achieves a sustained and controlled release of TQ, allowing for a greater stabilization of the terpene and a higher antimicrobial activity over time and offering a long-lasting, effective, and preservative effect in natural cosmetics. However, additional experiments are necessary to support the use of our nanoparticles in humans before commercialization.

\section{Materials and Methods}

Low molecular weight chitosan (CH) (50-190 kDa) with a 75-85\% degree of deacetylation, tripolyphosphate (TPP), 3-(4-dimethylthiazol-2-yl)-2 5-diphenyltetrazolium bromide (MTT), thymoquinone (TQ), and all the solvents were supplied by Sigma-Aldrich (Madrid, Spain). Microorganisms were purchased from the American Type Culture Collection (Manassas, VA, USA), namely E. coli (ATCC25922), P. aureginosa (ATCC27853), S. aureus (ATCC 6538), C. albicans (ATCC 10231), and A. brasiliensis (ATCC16404).

\subsection{Preparation of Loaded and Unloaded Thymoquinone-Chitosan Nanoparticles (NPCH-TQ) 4.1.1. Formulation of NPCH}

Chitosan nanoparticles (NPCH) were formulated through the ionic-gelation method described by [28]. Briefly, $\mathrm{CH}$ solution at $0.2 \%$ was prepared by dissolving $\mathrm{CH}$ flakes in acetic acid at $1 \%$ under continuous stirring overnight. Then, $50 \mathrm{~mL}$ of $\mathrm{CH}$ solution was mixed at $1000 \mathrm{RPM}$ in a $1 \%$ Tween 80 solution and heated to $50{ }^{\circ} \mathrm{C}$. Finally, TPP aqueous solution at $0.2 \%$ was added dropwise at $2 \mathrm{~mL} / \mathrm{min}$ under continuous stirring to induce the ionic gelation to form the nanoparticles. Afterward, agitation was carried out at 700 RPM for $40 \mathrm{~min}$. The nanoparticles were collected after centrifugation at 15,000 RPM for $20 \mathrm{~min}$ at $4^{\circ} \mathrm{C}$ and subsequently washed several times with $\mathrm{mQ}$ water. The nanoparticle suspension was frozen at $-80{ }^{\circ} \mathrm{C}$ and freeze-dried for $48 \mathrm{~h}$ at $-50{ }^{\circ} \mathrm{C}$ (LyoQuest-85/208 V $60 \mathrm{~Hz}$, Teslar).

\subsubsection{Formulation of NPCH-TQ}

Encapsulation of TQ into chitosan nanoparticles (NPCH-TQ) was formulated in a two-step process. Firstly, oil-in-water emulsification $(\mathrm{o} / \mathrm{w})$ was carried out, followed by the ionic gelation method as described above. Briefly, $50 \mathrm{~mL}$ of previously prepared $\mathrm{CH}$ solution was mixed at $1000 \mathrm{RPM}$ in a $1 \%$ Tween 80 solution and heated at $50{ }^{\circ} \mathrm{C}$. Subsequently, different amounts of terpene, previously preheated at $50{ }^{\circ} \mathrm{C}$ to form the liquid state to perform different ratios of $\mathrm{CH}$ :terpenes $(1: 0,1: 0.25,1: 0.5,1: 0.75$, and 1:1 w/w), were added dropwise under continuous agitation and emulsified at 1500 RPM during $10 \mathrm{~min}$ at room temperature. Finally, TPP aqueous solution at $0.2 \%$ was added dropwise at $2 \mathrm{~mL} \mathrm{~min}^{-1}$ under continuous stirring to induce the ionic gelation. Afterward, agitation was carried out at $700 \mathrm{RPM}$ for $40 \mathrm{~min}$. The nanoparticles were collected after centrifugation at 15,000 RPM for $20 \mathrm{~min}$ at $4{ }^{\circ} \mathrm{C}$ and subsequently washed several times with $\mathrm{mQ}$ water to eliminate unencapsulated terpenes. The nanoparticle suspension was frozen at $-80{ }^{\circ} \mathrm{C}$ and freeze-dried for $48 \mathrm{~h}$ at $-50{ }^{\circ} \mathrm{C}$ (LyoQuest-85/208 V $60 \mathrm{~Hz}$, Teslar).

4.2. Determination of Encapsulation Efficiency and Loading Efficiency of Thymoquinone-Chitosan Nanoparticles (NPCH-TQ)

Determination of Encapsulated TQ in Nanoparticles

To determine the total amount of TQ encapsulated into chitosan nanoparticles, $2 \mathrm{~mL}$ acetonitrile was added to $5 \mathrm{mg}$ of TQ-NPCH, and suspension was sonicated for $1 \mathrm{~h}$ at room temperature and left overnight with the solvent. Then, the supernatant was filtered 
through $0.45 \mu \mathrm{m}$ Millipore filter to measure in HPLC. The mobile phase employed was acetonitrile:water 70:30 and the amount of TQ was measured at $254 \mathrm{~nm}$ [39].

Loading capacity (LC) and encapsulation efficiency (EE) of terpenes were calculated according to the following equations (Equation (1), Equation (2)):

$$
\begin{aligned}
\mathrm{LC} \% & =(\text { weight of encapsulated terpene }(\mathrm{mg})) \div(\text { weight of total } \\
& \text { (terpene encapsulated }+ \text { scaffold weight })(\mathrm{mg})) \times 100
\end{aligned}
$$

$\mathrm{EE} \%=($ weight of encapsulated terpene $(\mathrm{mg})) \div($ weight of terpene feeding $(\mathrm{mg}))$

$$
\times 100
$$

\subsection{Instrumental Characterization of Nanoparticles}

\subsubsection{Particle Size Analysis}

Characterization of nano-formulations (size, zeta potential, and polydispersity index (PDI)) was determined by dynamic light scattering (DLS) using a Zetasizer (3000 HSM Malvern Ltd., Madrid, Spain) with the following specifications: chitosan refractive index (IR) of 1.700 , absorption index 0.010 , and water solvent RI: 1.33 , with a viscosity of $0.8872 \mathrm{cP}$. Measurements were performed in triplicate.

\subsubsection{Chemical Analysis of Nanoparticles}

$\mathrm{R}$ spectra were recorded on an attenuated total reflectance-Fourier transform infrared (ATR-FTIR) spectrophotometer (VARIAN 640-IR with a Pike Diamond/KRS-5 HS Performance Crystal Plate), and the main peaks were given in $\mathrm{cm}^{-1}$. ATR allows us to use the samples directly in a solid or liquid state without the need of $\mathrm{KBr}$ or Lugol's iodine matrix. Specifically, for NPCH and NPCH-TQ, $20 \mathrm{mg}$ of nanoparticles was powdered in a mortar, and the thin solid was placed on the diamond plate and pressed until a homogeneous pellet was obtained. For TQ, being liquid, a drop of approximately $200 \mu \mathrm{L}$ was placed on the plate, and the tip was placed in such a way that the surface tension of the drop covered the diamond plate homogeneously. 256 scans were acquired at an instrument resolution of $1 \mathrm{~cm}^{-1}$ over the spectral range between 650 and $4000 \mathrm{~cm}^{-1}$ owing to the frequency cutoff of the ATR-FTIR internal reflection element (IRE) used.

\subsubsection{Thermal Properties of Nanoparticles}

The thermal decomposition mechanisms were determined on a thermogravimetric analyzer (TGA Q20, TA Instruments) fitted with a standard platinum pan. The differential scanning calorimetry (DSC) experiments were carried out using a DSC Q50 system (TA Instruments) equipped with a standard aluminum pan with $10^{\circ} \mathrm{C} / \mathrm{min}$ increasing heat rate $\left(30-320^{\circ} \mathrm{C}\right)$ to investigate the thermal stability of pure TQ, NPCH, and NPCH-TQ. A sample of indium was used as reference. In all cases, samples of about $3 \mathrm{mg}$ were heated at a $10^{\circ} \mathrm{C} \mathrm{min}^{-1}$ rate under nitrogen atmosphere.

\subsubsection{Morphology Studies of Nanoparticles}

High-resolution electron microscope images of NPCH-TQ were obtained on a Jeol JEM 210 TEM microscope operating at $200 \mathrm{kV}$ and equipped with an Oxford Link EDS detector. The resulting images were analyzed using Digital Micrograph ${ }^{\mathrm{TM}}$ software from Gatan.

\subsection{In Vitro Release Studies of NPCH-TQ}

One milligram of lyophilized nanoparticles was sealed in a dialysis membrane (molecular weight cut off $3500 \mathrm{Da}$ ) and suspended in $10 \mathrm{~mL}$ of phosphate-buffered saline at optimum $\mathrm{pH}$ in $\mathrm{CPs}(\mathrm{pH} 6)$ in continuous stirring at $200 \mathrm{rpm}$ to ensure homogeneity. At certain intervals of incubation time at $37^{\circ} \mathrm{C}, 3 \mathrm{~mL}$ of release medium was removed to evaluate the amount of released terpene and replaced with $3 \mathrm{~mL}$ of fresh medium. The concentration of released TQ was determined with a spectrophotometer at wavelengths of $258 \mathrm{~nm}$. Terpene release was performed in triplicate. 


\subsection{2,2-Diphenyl-1-Picrylhydrazyl (DPPH) Radical Scavenging Activity}

FRS, free radical scavenging activity, was determined as described previously [5]. Briefly, $0.5 \mathrm{~mL}$ for each concentration $(1 \mathrm{mg} / \mathrm{mL}, 500 \mu \mathrm{g} / \mathrm{mL}, 250 \mu \mathrm{g} / \mathrm{mL}$, and $125 \mu \mathrm{g} / \mathrm{mL})$ of synthetized NPCH-CAR, NPCH-EUG, and free terpenes was mixed with $0.1 \mathrm{mM}$ ethanolic DPPH radical solution $(1.5 \mathrm{~mL})$ and then mixed and kept in the dark at room temperature for $30 \mathrm{~min}$. The absorbance of the solution was measured at $517 \mathrm{~nm}$. The FRS was calculated by $\%=(\mathrm{A} 0-\mathrm{A} 1 / \mathrm{A} 0) \times 100$, where $\mathrm{A} 0$ is absorbance at Time $=0$ and $\mathrm{A} 1$ is the absorbance after $30 \mathrm{~min}$.

\subsection{Antimicrobial Assay}

Different ratios of CH:TQ (1:0.25, 1:0.5, 1:0.75, and 1:1) were evaluated in order to perform the optimal formulation. For antimicrobial analysis, the NPCH:TQ ratio 1:1 was chosen to perform the assay because this ratio has better loading efficiency values than the other ratios (50.7\%) plus similar sizes and PDIs.

The antimicrobial activity and minimum inhibitory concentration (MIC) of terpenes and nanoparticles were tested against the most common pathogenic microorganisms in cosmetics and those that the UNI EN ISO 11930:2012 recommend for preservative efficacy evaluation. Antimicrobial activity of nanoparticles against P. aeruginosa, E. coli, S. aureus, A. brasiliensis, and C. albicans was tested using the broth microdilution method [17,40]. Stock cultures were prepared from Culti-Loops ${ }^{\mathrm{TM}}$ (Sigma-Aldrich, Madrid, Spain) in Nutrient Broth (NB) and Potato Dextrose Broth (PDB) at $37^{\circ} \mathrm{C}$. Standardized inoculum was then created by dilution in Müller-Hinton medium to a final density of $0.5 \mathrm{McF}$ arland units by densitometer McFarland type DEN-1B (Biosan, Riga, Latvia). TQ, NPCH-TQ, and NPCH were tested in concentrations of $1000 \mu \mathrm{g} / \mathrm{mL}$ to $15.6 \mu \mathrm{g} / \mathrm{mL}$. Gentamicin (for bacteria) and Tebuconazole (for mold and yeast) were used as standards. After treatment, plates were incubated $24 \mathrm{~h}$ at $37^{\circ} \mathrm{C}$ for bacteria and $48 \mathrm{~h}$ at $30^{\circ} \mathrm{C}$ for yeast and fungi.

\subsection{Moisturizing Cream Formulation}

Two types of moisturizing creams were formulated: control cream without any preservative agent and NPCH-TQ with the addition of nanoparticles on formulated cream. The composition of both creams is shown in Table 3 Oil phase (B) and water phase (A) were preheated at $70{ }^{\circ} \mathrm{C}$ to achieve the fusion of oils and waxes present in this phase. Then, $\mathrm{B}$ was added slowly under agitation in a homogenizer at 3000 RPM to form an oil/water emulsion. Upon cooling, the thermolabile compounds (C) and preservatives (NPCH-TQ) at a dose of $500 \mu \mathrm{g} / \mathrm{mL}$ (maximum of MIC value) were added to the cooled cream under continuous agitation.

\subsection{Preservative Activity of AgNPs in Formulated Cream}

The preservative efficacy of NPCH-TQ in the moisturizing creams was carried out comparing the antimicrobial activity of NPCH-TQ cream against non-preserved cream. Briefly, twenty grams of each cream (Control and NPCH-TQ) was diluted with a sterile $\mathrm{NaCl}$ solution at $0.9 \%$. Then, each cream was inoculated with $10^{5} \mathrm{UFC} / \mathrm{mL}$ for bacteria, $10^{4} \mathrm{CFU} / \mathrm{mL}$ for yeast, and $10^{3} \mathrm{CFU} / \mathrm{mL}$ for mold. Contaminated creams were stored at room temperature for 30 days. After 2, 7, 14, and 28 days, one gram of each contaminated cream was diluted and spread in Petri dishes to count CFUs of bacteria. Yeast and mold contaminations were evaluated at 7, 14, and 28 days using the same methodology.

Supplementary Materials: The following supporting information can be downloaded at: https: //www.mdpi.com/article/10.3390/ijms23020898/s1.

Author Contributions: Conceptualization, E.N. and O.A.; methodology, M.M.-L. and J.C.G.M.; software, A.J.L.-J.; validation, E.N., O.A., and L.G.-G.; formal analysis and investigation, E.N.; writing-original draft preparation, E.N.; writing—review and editing, O.A.; supervision, E.N., O.A., and L.G.-G.; project administration, O.A.; funding acquisition, L.G.-G. All authors have read and agreed to the published version of the manuscript. 
Funding: This research was funded by the University of Castilla-La Mancha (UCLM, Spain), grant number 2020-GRIN-28857.

Institutional Review Board Statement: Not applicable.

Informed Consent Statement: Not applicable.

Data Availability Statement: Not applicable.

Acknowledgments: Our thanks to Javier Argandoña for his technical assistance and to K.A. Walsh for language revision.

Conflicts of Interest: The authors declare no conflict of interest.

Sample Availability: Samples of the compounds are not available from the authors.

\section{References}

1. Michalek, I.; John, S.; Dos Santos, F.C. Microbiological contamination of cosmetic products-Observations from Europe, 2005-2018. J. Eur. Acad. Dermatol. Venereol. 2019, 33, 2151-2157. [CrossRef]

2. Halla, N.; Fernandes, I.P.; Heleno, S.A.; Costa, P.; Boucherit-Otmani, Z.; Boucherit, K.; Rodrigues, A.E.; Ferreira, I.C.F.R.; Barreiro, M.F. Cosmetics Preservation: A Review on Present Strategies. Molecules 2018, 23, 1571. [CrossRef]

3. Palacios, S.; Shaman, F.; Garcá, J.A. Prevalence of cosmetic sensitivity among beauticians. Allergol. Immunopathol. 1995, 23, 148-152.

4. Nowak, K.; Jabłońska, E.; Ratajczak-Wrona, W. Controversy around parabens: Alternative strategies for preservative use in cosmetics and personal care products. Environ. Res. 2021, 198, 110488. [CrossRef] [PubMed]

5. Mondéjar-López, M.; López-Jiménez, A.; Abad-Jordá, M.; Rubio-Moraga, A.; Ahrazem, O.; Gómez-Gómez, L.; Niza, E. Biogenic Silver Nanoparticles from Iris tuberosa as Potential Preservative in Cosmetic Products. Molecules 2021, 26, 4696. [CrossRef] [PubMed]

6. Wang, J.; Liu, Y.; Kam, W.R.; Li, Y.; Sullivan, D.A. Toxicity of the cosmetic preservatives parabens, phenoxyethanol and chlorphenesin on human meibomian gland epithelial cells. Exp. Eye Res. 2020, 196, 108057. [CrossRef]

7. De Matos, S.P.; Teixeira, H.F.; De Lima, Á.A.; Veiga-Junior, V.F.; Koester, L.S. Essential Oils and Isolated Terpenes in Nanosystems Designed for Topical Administration: A Review. Biomolecules 2019, 9, 138. [CrossRef]

8. Aslam, H.; Shahzad, M.; Shabbir, A.; Irshad, S. Immunomodulatory effect of thymoquinone on atopic dermatitis. Mol. Immunol. 2018, 101, 276-283. [CrossRef]

9. Darakhshan, S.; Pour, A.B.; Colagar, A.H.; Sisakhtnezhad, S. Thymoquinone and its therapeutic potentials. Pharmacol. Res. 2015, 95-96, 138-158. [CrossRef]

10. Mahmoud, Y.K.; Abdelrazek, H.M. Cancer: Thymoquinone antioxidant/pro-oxidant effect as potential anticancer remedy. Biomed. Pharmacother. 2019, 115, 108783. [CrossRef]

11. Kalam, M.A.; Raish, M.; Ahmed, A.; Alkharfy, K.M.; Mohsin, K.; Alshamsan, A.; Al-Jenoobi, F.I.; Al-Mohizea, A.M.; Shakeel, F. Oral bioavailability enhancement and hepatoprotective effects of thymoquinone by self-nanoemulsifying drug delivery system. Mater. Sci. Eng. C Mater. Biol. Appl. 2017, 76, 319-329. [CrossRef] [PubMed]

12. Magdy, M.-A.; Hanan, E.-A.; Nabila, E.-M. Thymoquinone: Novel gastroprotective mechanisms. Eur. J. Pharmacol. 2012, 697, 126-131. [CrossRef] [PubMed]

13. Shaterzadeh-Yazdi, H.; Noorbakhsh, M.-F.; Samarghandian, S.; Farkhondeh, T. An Overview on Renoprotective Effects of Thymoquinone. Kidney Dis. 2018, 4, 74-82. [CrossRef]

14. Tabeshpour, J.; Mehri, S.; Abnous, K.; Hosseinzadeh, H. Neuroprotective Effects of Thymoquinone in Acrylamide-Induced Peripheral Nervous System Toxicity Through MAPKinase and Apoptosis Pathways in Rat. Neurochem. Res. 2019, 44, 1101-1112. [CrossRef]

15. Abdel-Daim, M.M.; El-Ela, F.I.A.; Alshahrani, F.K.; Bin-Jumah, M.; Al-Zharani, M.; Almutairi, B.; Alyousif, M.S.; Bungau, S.; Aleya, L.; Alkahtani, S. Protective effects of thymoquinone against acrylamide-induced liver, kidney and brain oxidative damage in rats. Environ. Sci. Pollut. Res. 2020, 27, 37709-37717. [CrossRef]

16. Fan, Q.; Yuan, Y.; Jia, H.; Zeng, X.; Wang, Z.; Hu, Z.; Gao, Z.; Yue, T. Antimicrobial and anti-biofilm activity of thymoquinone against Shigella flexneri. Appl. Microbiol. Biotechnol. 2021, 105, 4709-4718. [CrossRef]

17. Niza, E.; Božik, M.; Bravo, I.; Clemente-Casares, P.; Sánchez, A.L.; Juan, A.; Klouček, P.; Alonso-Moreno, C. PEI-coated PLA nanoparticles to enhance the antimicrobial activity of carvacrol. Food Chem. 2020, 328, 127131. [CrossRef]

18. Kamkar, A.; Molaee-Aghaee, E.; Khanjari, A.; Akhondzadeh-Basti, A.; Noudoost, B.; Shariatifar, N.; Sani, M.A.; Soleimani, M Nanocomposite active packaging based on chitosan biopolymer loaded with nano-liposomal essential oil: Its characterizations and effects on microbial, and chemical properties of refrigerated chicken breast fillet. Int. J. Food Microbiol. 2021, $342,109071$. [CrossRef] [PubMed]

19. Sani, M.A.; Tavassoli, M.; Hamishehkar, H.; McClements, D.J. Carbohydrate-based films containing pH-sensitive red barberry anthocyanins: Application as biodegradable smart food packaging materials. Carbohydr. Polym. 2021, 255, 117488. [CrossRef] 
20. Tavassoli, M.; Sani, M.A.; Khezerlou, A.; Ehsani, A.; McClements, D.J. Multifunctional nanocomposite active packaging materials: Immobilization of quercetin, lactoferrin, and chitosan nanofiber particles in gelatin films. Food Hydrocoll. 2021, 118, 106747. [CrossRef]

21. Lisuzzo, L.; Cavallaro, G.; Parisi, F.; Milioto, S.; Fakhrullin, R.; Lazzara, G. Core/Shell Gel Beads with Embedded Halloysite Nanotubes for Controlled Drug Release. Coatings 2019, 9, 70. [CrossRef]

22. Potaś, J.; Szymańska, E.; Basa, A.; Hafner, A.; Winnicka, K. Tragacanth Gum/Chitosan Polyelectrolyte Complexes-Based Hydrogels Enriched with Xanthan Gum as Promising Materials for Buccal Application. Materials 2020, 14, 86. [CrossRef] [PubMed]

23. Szymańska, E.; Woś-Latosi, K.; Jacyna, J.; Dąbrowska, M.; Potaś, J.; Markuszewski, M.J.; Winnicka, K. The Correlation between Physical Crosslinking and Water-Soluble Drug Release from Chitosan-Based Microparticles. Pharmaceutics 2020, 12, 455. [CrossRef] [PubMed]

24. Ince, I.; Yıldırım, Y.; Güler, G.; Medine, E.I.; Ballıca, G.; Kusdemir, B.C.; Göker, E. Synthesis and characterization of folic acidchitosan nanoparticles loaded with thymoquinone to target ovarian cancer cells. J. Radioanal. Nucl. Chem. 2020, $324,71-85$. [CrossRef]

25. Fahmy, H.M.; Khadrawy, Y.A.; Daim, T.M.A.-E.; Elfeky, A.S.; Rabo, A.A.A.; Mustafa, A.B.; Mostafa, I.T. Thymoquinoneencapsulated chitosan nanoparticles coated with polysorbate 80 as a novel treatment agent in a reserpine-induced depression animal model. Physiol. Behav. 2020, 222, 112934. [CrossRef]

26. Aranaz, I.; Acosta, N.; Civera, C.; Elorza, B.; Mingo, J.; Castro, C.; Gandía, M.D.L.L.; Caballero, A.H. Cosmetics and Cosmeceutical Applications of Chitin, Chitosan and Their Derivatives. Polymers 2018, 10, 213. [CrossRef]

27. Estupiñán, Ó.; Niza, E.; Bravo, I.; Rey, V.; Tornín, J.; Gallego, B.; Clemente-Casares, P.; Moris, F.; Ocaña, A.; Blanco-Lorenzo, V.; et al. Mithramycin delivery systems to develop effective therapies in sarcomas. J. Nanobiotechnol. 2021, 19, 267. [CrossRef]

28. Mondéjar-López, M.; Rubio-Moraga, A.; López-Jimenez, A.J.; Martínez, J.C.G.; Ahrazem, O.; Gómez-Gómez, L.; Niza, E. Chitosan nanoparticles loaded with garlic essential oil: A new alternative to tebuconazole as seed dressing agent. Carbohydr. Polym. 2021, 277, 118815. [CrossRef]

29. Tullio, V.; Nostro, A.; Mandras, N.; Dugo, P.; Banche, G.; Cannatelli, M.; Cuffini, A.M.; Alonzo, V.; Carlone, N. Antifungal activity of essential oils against filamentous fungi determined by broth microdilution and vapour contact methods. J. Appl. Microbiol. 2007, 102, 1544-1550. [CrossRef]

30. Dehghani, H.; Hashemi, M.; Entezari, M.; Mohsenifar, A. The Comparison of Anticancer Activity of Thymoquinone and Nanothymoquinone on Human Breast Adenocarcinoma. Iran. J. Pharm. Res 2015, 14, 539-546. [PubMed]

31. Alam, S.; Mustafa, G.; Khan, Z.I.; Islam, F.; Bhatnagar, A.; Ahmad, F.J.; Kumar, M. Development and evaluation of thymoquinoneencapsulated chitosan nanoparticles for nose-to-brain targeting: A pharmacoscintigraphic study. Int. J. Nanomed. 2012, 7, 5705-5718. [CrossRef] [PubMed]

32. Woranuch, S.; Yoksan, R. Eugenol-loaded chitosan nanoparticles: I. Thermal stability improvement of eugenol through encapsulation. Carbohydr. Polym. 2013, 96, 578-585. [CrossRef]

33. Ahmad, R.; Kaus, N.H.M.; Hamid, S. Synthesis and Characterization of PLGA-PEG Thymoquinone Nanoparticles and Its Cytotoxicity Effects in Tamoxifen- Resistant Breast Cancer Cells. In Cancer Biology and Advances in Treatment. Advances in Experimental Medicine and Biology; Pham, P.V., Ed.; Springer: Cham, Switzerland, 2018; Volume 1292.

34. Bhatta, A.; Krishnamoorthy, G.; Marimuthu, N.; Dihingia, A.; Manna, P.; Biswal, H.T.; Das, M.; Krishnamoorthy, G. Chlorin e6 decorated doxorubicin encapsulated chitosan nanoparticles for photo-controlled cancer drug delivery. Int. J. Biol. Macromol. 2019, 136, 951-961. [CrossRef] [PubMed]

35. Upadhyay, P.; Sarker, S.; Ghosh, A.; Gupta, P.; Das, S.; Ahir, M.; Bhattacharya, S.; Chattopadhyay, S.; Ghosh, S.; Adhikary, A. Transferrin-decorated thymoquinone-loaded PEG-PLGA nanoparticles exhibit anticarcinogenic effect in non-small cell lung carcinoma via the modulation of miR-34a and miR-16. Biomater. Sci. 2019, 7, 4325-4344. [CrossRef] [PubMed]

36. Anand, T.; Anbukkarasi, M.; Thomas, P.A.; Geraldine, P. A comparison between plain eugenol and eugenol-loaded chitosan nanoparticles for prevention of in vitro selenite-induced cataractogenesis. J. Drug Deliv. Sci. Technol. 2021, 65, 102696. [CrossRef]

37. Chen, F.; Shi, Z.; Neoh, K.; Kang, E. Antioxidant and antibacterial activities of eugenol and carvacrol-grafted chitosan nanoparticles. Biotechnol. Bioeng. 2009, 104, 30-39. [CrossRef]

38. Randhawa, M.A.; Gondal, M.A.; Al-Zahrani, A.-H.J.; Rashid, S.G.; Ali, A. Synthesis, morphology and antifungal activity of nanoparticulated amphotericin-B, ketoconazole and thymoquinone against Candida albicans yeasts and Candida biofilm. J. Environ. Sci. Health Part A 2015, 50, 119-124. [CrossRef]

39. Xiao, X.-Y.; Zhu, Y.-X.; Bu, J.-Y.; Li, G.-W.; Zhou, J.-H.; Zhou, S.-P. Evaluation of Neuroprotective Effect of Thymoquinone Nanoformulation in the Rodent Cerebral Ischemia-Reperfusion Model. BioMed Res. Int. 2016, 2016, 2571060. [CrossRef]

40. Qidwai, A.; Kumar, R.; Dikshit, A. Green synthesis of silver nanoparticles by seed of Phoenix sylvestris L. and their role in the management of cosmetics embarrassment. Green Chem. Lett. Rev. 2018, 11, 176-188. [CrossRef] 